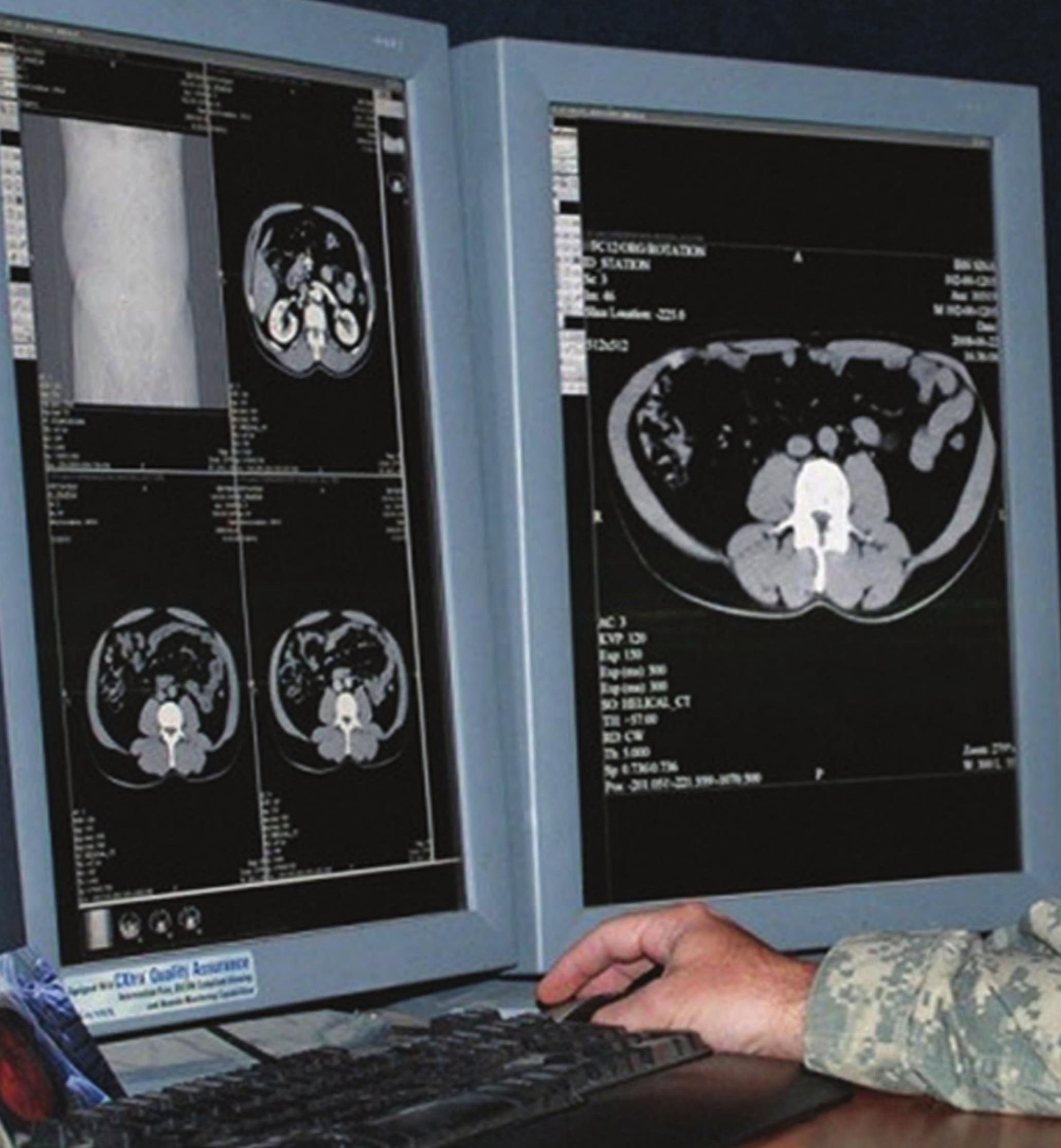




\begin{tabular}{|c|c|c|}
\hline $\begin{array}{l}\Omega \\
\text { Carme Hernandez', } \\
\text { Jennifer Mallow }{ }^{2} \text {, } \\
\text { Georgia L. } \\
\text { Narsavage }^{3}\end{array}$ & $\begin{array}{l}\text { DIn } \\
\text { Integrated Care Unit, Hospital } \\
\text { Clinic, Barcelona Spain } \\
{ }^{2} \text { School of Nursing and West Virginia } \\
\text { Clinical Translational Science } \\
\text { Institute (WVCSSI), West Virginia } \\
\text { University (WVU), Morgantown, WV, } \\
\text { USA } \\
{ }^{3} \text { Robert C. Byrd Health Sciences } \\
\text { Center, Mary Babb Randolph Cancer } \\
\text { Center, and WVCTSI, West Virginia } \\
\text { University, Morgantown, WV, USA }\end{array}$ & $\begin{array}{l}\equiv \\
\text { Georgia L. Narsavage, West gnarsavage@hsc.wvu.edu } \\
\text { Virginia University, 3602 } \\
\text { Health Sciences Center, } \\
\text { P.O. Box 9600, } \\
\text { Morgantown, WV } \\
\text { 26506-9600, USA }\end{array}$ \\
\hline
\end{tabular}

\section{Delivering telemedicine interventions in chronic respiratory disease}

\section{Educational Aims}

- To explain the basic principles of telemedicine applicable to chronic respiratory diseases

- To review telemedicine interventions for patients with chronic respiratory diseases

- To outline the advantages and limitations (including cost and barriers to implementation) of telemedicine for patients with chronic respiratory diseases

- To propose recommendations for clinical management of patients receiving telemedicine for chronic respiratory diseases

\section{The potential of ICT for respiratory patient care}

Information and communication technologies (ICT) have great potential to support organisational changes for enhancing chronic care management. Neither ICT alone nor monitoring by itself can drive successful outcomes for respiratory patient care. However, ICT has been shown to be effective as a support for professionals to improve care. The chronic care model using integrated care services (ICS) with the support of ICT has demonstrated potential to improve respiratory patient care.

The World Health Organization (WHO), in establishing the Global Observatory for eHealth, has developed a disease-oriented definition of telemedicine: "The delivery of healthcare services, where distance is a critical factor, by all health professionals using ICT for the exchange of valid information for diagnosis, treatment and prevention of disease and injuries, research and evaluation, and for the continuing education of healthcare providers, all in the interest of advancing the health of individuals and their
Statement of Interest G.L. Narsavage reports $\mathrm{NIH}$ funding received for telemonitoring research (as a PI with Co-PI Y-J Chen). NIH/ $\mathrm{NCI}$ R15-AREA (ARRA). "Pilot: Home

Telemonitoring for SelfManagement Education of Patients with Lung Cancer" (1R15CA150999-01) 2010-2013 (\$366,250). J. Mallow was supported by the WVCTSI through the National Institute of General Medical NIH/NIGMS Award Number U54GM104942.

HERMES syllabus link: module B.1.1, B.1.4, B.5.2, B.5.3, B.15.1, E.1.12, F.6 
communities" [1]. In addition to traditional informational technologies (IT) activities, ICT today includes services such as telephone transmission with or without wires, other broadcast media and multiple methods of providing audio-visual transmission of information [2]. A more recent definition states that telemedicine "seeks to improve a patient's health by permitting two-way, realtime interactive communication between the patient, and the physician or practitioner at the distant site. This electronic communication means the use of interactive telecommunications equipment that includes, at a minimum, audio and video equipment" [3].

Support tools and systems designed to assist clinical decisions using research evidence, guidelines and patient information, remotely accessed using electronic health records and web-based portals are available across the globe. Patient-level and population-level clinical and administrative data can be accessed at a distance by clinicians to provide healthcare and can be easily stored and managed. These services, known as "telemedicine" or "telehealth" have been used interchangeably and involve secure transmission of medical and other healthcare data and information as text, sound, images or other media as needed for the prevention, diagnosis and treatment of disease and the follow-up of patients. This article will use both terms as appropriate to health and disease care. Biometric devices, such as equipment measuring heart rate, blood pressure, forced spirometry and symptom diaries, can be used remotely to monitor and manage patients with chronic respiratory diseases (CRDs). Telemedicine can transform the delivery of healthcare services by migrating healthcare delivery away from hospitals and into patient homes [4].

Telemedicine services have been classified into two broad categories: 1) professional-level telemedicine occurring between health professionals (e.g. clinician-to-clinician, doctor-todoctor, nurse-to-doctor), including teleconsultation, teleradiology and telepathology; and 2) professional-patient care between clinicians and patients including telemonitoring, telehomecare, assisted-emergency care (e.g. for emergency medical technician support) and internet-based consultations. Professionallevel interactions may include advice or case conferencing, as well as remote access to the patients' health records and support among clinicians about the case. Professional-patient communication through telemedicine promotes the use of self-care at home, through self-monitoring and education with support from the professional. The remote monitoring of the patient allows for the collection of routine information on the patient's health status outside of the locale of the clinician, i.e. at a distance from their office or hospital. Telemedicine offers a two-way transfer of information as evidence-based care can be supported using inbuilt clinical pathways, electronic protocols and guidelines and can subsequently be used as a foundation for policy making based on the data collected before, during and after each care episode.

\section{Chronic conditions, comorbidity and telemonitoring}

Helping people decrease their risk for chronic diseases and improving a patient's ability to live with chronic conditions are international concerns. The increasing incidence of chronic conditions is approaching epidemic levels and finding evidence-based and effective strategies to promote health and for the prevention and management of these conditions is essential. Multiple studies have demonstrated that transforming care for this population requires a fundamental shift towards care that is coordinated around a range of services [5]. Using models that provide a structure to healthcare for individuals with chronic diseases and comorbidities, such as the chronic care model, within an integrated care system has great potential to improve patient care and outcomes [6].

Patients suffering with multiple chronic conditions are particularly vulnerable to suboptimal quality care and coordination of care is more difficult in patients with multiple chronic conditions taking more medication who are more likely to suffer adverse drug events. Comorbidities with respiratory diseases have a high impact on hospital admissions. The presence of comorbidity influences healthcare decisions, including the prevention of disease, complexity of treatment and service utilisation, as well as influencing outcomes, such as activity limitations, participation restriction and mortality. Multiple chronic conditions also make it more challenging for 
patients to effectively engage in self-care. For chronically ill patients, it is estimated that $90 \%$ of the care must be self-managed or managed in coordination with the healthcare system without burdening it. HeRnANDEZ et al. [7] provided an overview of care coordination and its challenges pointing to the potential for telemedicine in patients with chronic conditions, including respiratory diseases.

Improving the care of chronic respiratory patients with multiple chronic conditions is indeed a challenge. Care delivery must include prevention, early diagnosis, therapeutic education empowerment to maintain health and assistance in managing their chronic diseases. Telemedicine can help individuals acquire the skills to manage their health and maintain an active life as well as enhance health-service capacity due to its accessibility and increasing acceptance by both providers and patients with CRDs [8]. Targeted web pages have also helped to improve health behaviours and raise awareness about healthy living.

Effective CRD management, with appropriate coordination and monitoring of care, can assist in reducing the long-term consequences of respiratory diseases and reduce the impact of chronic conditions [9,10]. Home telemonitoring of chronic diseases seems to be a promising patient-management approach, which is able to produce accurate and reliable data as well as empower patients by influencing their behaviour to eventually potentially improve their condition. By incorporating self-management practices and appropriate telemedicine case management, the use of emergency care and unplanned hospital admissions/readmissions have been reduced $[9,10]$.

\section{Types of telemedicine systems}

\section{Interactive telemedicine services}

Interactive telemedicine services offer concurrent interactions between patients and clinicians/doctors. This service includes telephone and web-based communication as well as home visits. An example of the basic components of a telemedicine system is shown in figure 1 .

\section{Remote monitoring}

Remote monitoring allows clinicians and doctors to check a patient remotely using varied telemedicine equipment through self-monitoring/testing systems. "Telecare" (a combination of alarms and sensors) or "telehealth" (equipment for monitoring physiological signs and symptoms, such as spirometers) may be used.

\section{Store-and-forward telemedicine}

Store-and-forward telemedicine involves the transmission of data regarding the disease, such as medical images, biological readings, etc., to a medical specialist for evaluation offline. This type of telemedicine is asynchronous, meaning that both parties do not need to be online at the same time.

\section{Specialist and primary-care consultations}

Specialist and primary-care consultations involve patient and doctor and/or other professionals interactions using live video technology or through sending diagnostic media and patient data to a specialist for assessment.

\section{Imaging services}

Imaging services (e.g. radiology) continue to create protocols for maximum use of telemedicine from remote locations with thousands of images "read" or interpreted by specialist providers annually.

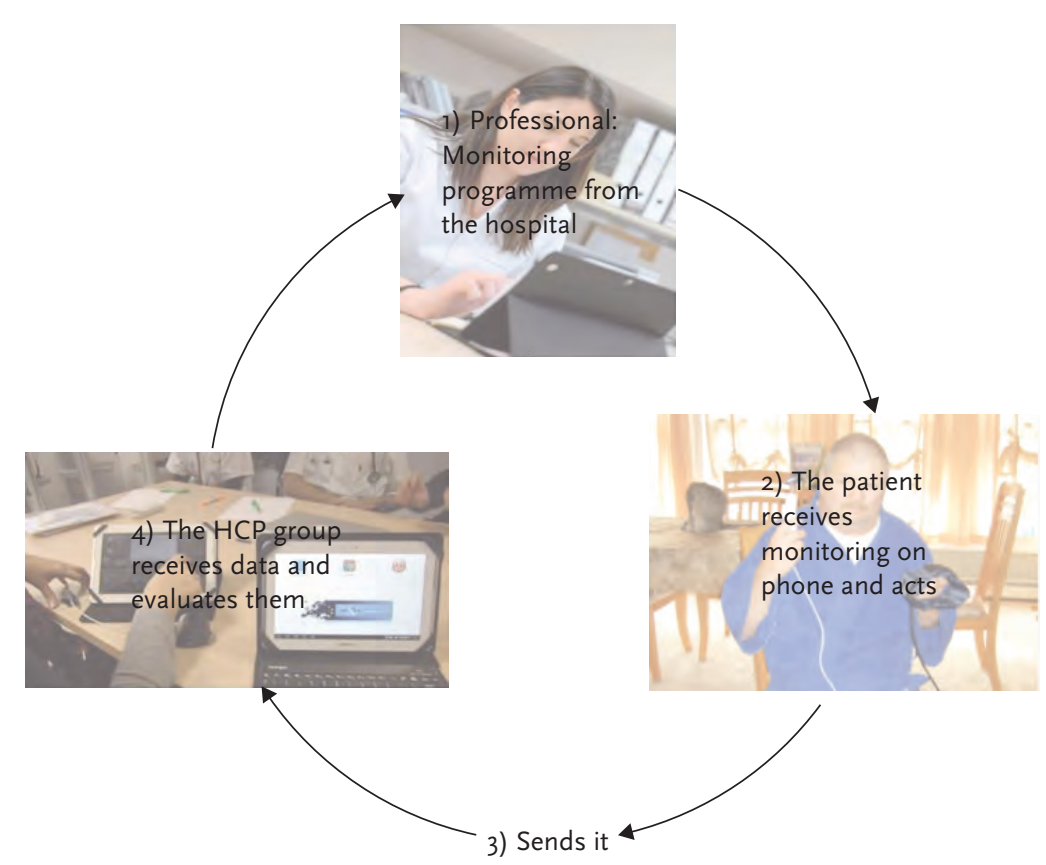

Figure 1

Basic components of a telemedicine system. 


\section{Types of interventions}

\section{Telephone counselling}

Although not a highly technical form of telemedicine, telephone counselling for smoking cessation was an early intervention reviewed by the Cochrane Collaboration [11]. The review concluded that proactive telephone counselling helps smokers who seek it from "quitlines". Telephone quitlines provide important access to support for smokers, and the use of call-back counselling makes them even more useful. There are, however, limited data regarding how many calls are optimal; additionally, in settings other than smoking cessation, whether there is benefit to this proactive telephone counselling. There appears to be a dose response, with one or two brief calls being less likely to provide a measurable benefit; however, three or more calls increases the cessation success compared with a minimal intervention in the form of standard self-help materials or brief advice, or with pharmacotherapy alone. Current evidence has also shown a benefit of smoking cessation interventions on long-term outcomes through the use of mobile telephones (predominantly text messaging), though results were heterogeneous, with three out of five studies crossing the line of no effect. More research is required into other forms of mobile telephone-based interventions for smoking cessation, including cost effectiveness, in other contexts such as low income countries.

\section{Forced spirometry}

The challenge of early diagnosis and management of CRD is seen in those with greater prevalence, like chronic obstructive pulmonary disease (COPD) and asthma. Forced spirometry is important for diagnosis and management of respiratory diseases. A webbased application was tested for development of high-quality spirometry skills in community/primary-care settings. BURGOS et al. [12] examined the efficacy, acceptability and usability of a web-based application covering three main functions: 1) accessibility to educational material for continuous professional development; 2) remote support for quality assurance of tests performed by nonexperts; and 3) remote assistance for lung function interpretation. This research indicated sustained benefit of online intervention by increasing high-quality spirometry tests, and professionals acknowledged the usefulness of a web-based tool for remote assistance with interpretation of the results as well as to increase non-expert professionals' skills for performing high-quality forced spirometry in primary care. The study expands the potential of primary care for the diagnosis and management of patients with pulmonary diseases.

\section{Computed tomography and diagnostic support}

A chest computed tomography (CT) scan, a painless noninvasive test, is often used to follow-up chest radiography to diagnose a respiratory problem or to identify the cause of exacerbation of lung symptoms, such as increasing shortness of breath [13]. Understanding and interpreting CT scans requires specialised training, and teleradiology offers the ability for healthcare providers to send CT images from one location to another [14]. In order to use teleradiology for CT review, three things are needed: 1) access to a CT scan machine; 2) a transmission network in order to securely send patient CT images; and 3) a receiving computer with a high-quality display screen for reviewing the image. This allows the local doctor to stay with the patient, and can be cost-effective in specialist clinicians' practices.

Integrated care programmes for chronic respiratory patients through the employment of advanced nurses and other care providers

Delivering healthcare to populations with limited access to care and a high level of frailty are some of the most advantageous aspects of telemedicine. Figure 2 shows multiple functional areas for the technological platforms used in telemedicine. A call centre and a web-based application used in the integrated care unit in the Clinic Hospital, Barcelona, Spain, for example, facilitated patient accessibility and interactions among professionals working at different levels of the system. Advanced practice nurses (APRNs) have been leading the movement to provide quality, affordable and timely care to patients, in systems that face the challenge of limited numbers of healthcare providers [15]. As most APRNS are care providers, using APRNs in a telemedicine initiative is common. Studies 


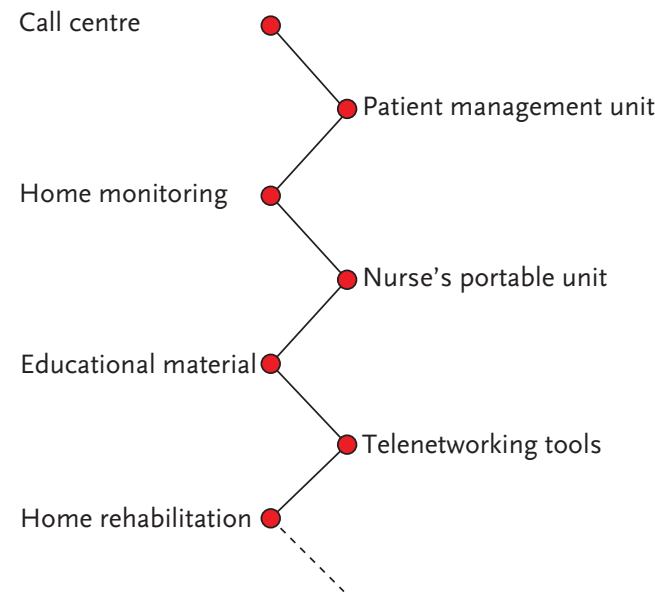

Figure 2

Telemedicine technological platform: functional areas.

that have examined telemedicine in recent years have supported the feasibility of the APRN role $[16,17]$. Types of telemedicine that have been used by nurses can be as low-tech as providing advice, education and test results over the telephone; however, as technology advances, APRNs are using mobile devices including phones and tablets, video communication and wearable Bluetooth-enabled patient self-monitoring systems to deliver healthcare remotely [18]. While the use of telemedicine has enabled APRNs to provide care across geographical distances, the practice of APRNs is bound by a state-based licensing system within the USA and some European countries, such that the ability to practice across borders may be prohibited; a policy barrier needing resolution.

\section{Monitoring health status and making associated care decisions}

The use of telemedicine can enhance clinical decision-making related to patient care. Standard practice guidelines for the amount of self-management activity and changes to individual care plans based on these readings parallel traditional care; however, the lack of clarity in supporting clinician decision-making may be, in large part, why healthcare professionals are often averse or indifferent to adopting telemedicine applications [19]. Decisions related to the extension of the most promising telemedicine applications are complex and should include multiple stakeholders such as practitioners, healthcare regulators and governments [20]. Key elements for monitoring patients with CRD via telemedicine are shown in figure 3 .

\section{Telemedicine and asthma}

Asthma is one of the most prevalent noncommunicable chronic respiratory conditions [21], occurring throughout the lifespan. More than $14 \%$ of children have been diagnosed with asthma [21]. However, asthma is underdiagnosed and under-treated [22, 23]. Underdiagnosis and ineffective therapy are major factors in morbidity and mortality [24]. Uncontrolled breathlessness/wheezing, leading to significant morbidity and mortality, often occurs in low- and lower-middle income countries [24]

Management of asthma requires an accurate diagnosis, assessment and monitoring of interventions and responses, education, controlling environmental factors and pharmacological therapy [25]. Medication and avoiding asthma triggers can reduce the severity of asthma and enable individuals to have a good quality of life [26]. Targeted management using best-practice guidelines and ongoing patient education and support are needed.

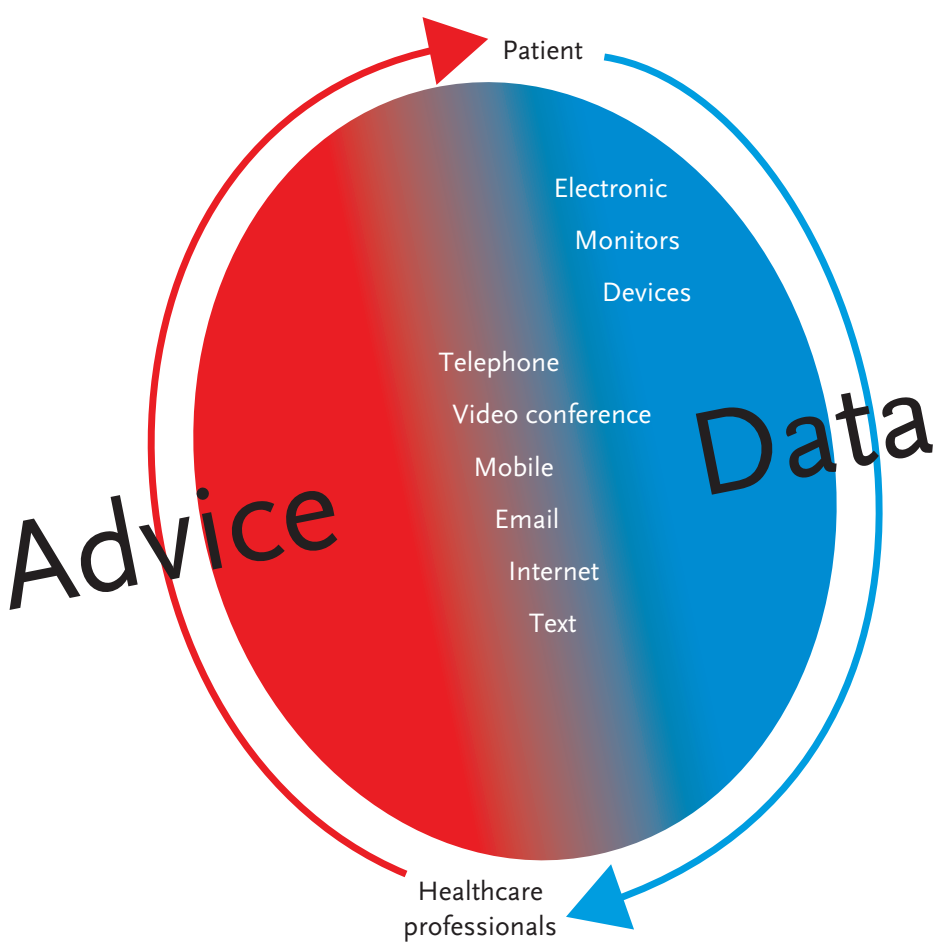

Figure 3

Key elements for chronic respiratory disease management via telemedicine. 


\section{Description of telemedicine interventions for patients with asthma}

The use of telehealthcare in asthma management is complex and has been offered in multiple ways including through patient education and counselling, replacing face-toface nursing/physician visits, sending reminders regarding adherence to medications and other treatment regimens and the remote monitoring of patients' health parameters [27]. The purpose of these telehealth interventions is to enable early detection of disease exacerbation, provide timely intervention for early symptom management, reduce unscheduled visits to the emergency room and prevent hospitalisations [27]. RYAN et al. [28] compared paper-based monitoring to twice-daily mobile telephone-based transmission of symptoms, drug use and peak flow with immediate feedback prompting actions according to an agreed plan for asthma care. However, the mobile telephone-based model of monitoring did not result in any clinical advantages over paper-based care when guideline-standard clinical support services were provided to both groups, but clinically relevant improvements were seen in both groups, suggesting that the telemonitoring was not the crucial ingredient in improving outcomes; however, the mobile telephonebased model of asthma care was more expensive than the paper-based model. A major limitation of this study was the lack of control for severity of asthma. A systematic meta-analysis of telehealth for asthma interventions revealed interventions were unlikely to result in clinically relevant improvements in health outcomes in those with relatively mild asthma, but they may have a role in managing those with more severe disease who are at high risk of hospital admission. The lack of an efficacy comparison between the different types of telehealth interventions point to the need for further trials, evaluating the comparative effectiveness of telemedicinebased educational interventions, pulmonary function interventions, interactive telemedicine and remote monitoring with costeffectiveness for the wide range of telehealth interventions [27].

\section{Telemedicine and COPD}

The European Commission has recognised the potential of telemedicine for monitoring patients from their homes in the management of COPD [29]. COPD, including emphysema and bronchitis, consists of airflow blockage that restricts breathing and negatively impacts functioning. Tobacco smoke, exposure to air pollutants in the home and workplace, genetics and respiratory infections are all factors in the development and progression of COPD worldwide. The WHO estimates that 65 million people have moderate-to-severe COPD. More than $50 \%$ of adults with low pulmonary function are not aware that they have COPD and one in 20 deaths worldwide ( $>3$ million people) are related to COPD with approximately $90 \%$ of COPD-related deaths occurring in low- and middle-income countries.

Management of COPD is multi-faceted in order to assess and monitor the disease, reduce risk factors, such as smoking and air pollution, stabilise care to prevent disease progression and manage exacerbations and comorbid conditions. Treatment focuses on monitoring changes and relieving symptoms, improving function/exercise tolerance and ongoing vigilance [7]. Targeted management using best-practice guidelines (patient oriented) and ongoing patient education and support are needed with adaptation to comorbid conditions. Teleassistance has been shown to prevent hospitalisation as well as to be cost-effective in patients on oxygen or home mechanical ventilation. Teleassistance has been well accepted by patients with CRD and caregivers [30].

\section{Description of telemedicine interventions for patients with COPD}

A multi-parametric remote monitoring system reduced the rate of COPD exacerbations [31] in a randomised clinical trial of 100 COPD patients in Global Initiative for Chronic Obstructive Lung Disease (GOLD) stage IIIII, aged $\geqslant 65$ years over a 9-month period. The intervention group measured oxygen saturation, heart rate, temperature and physical activity using a telemedicine commercial cellular phone plus specific software. Realtime outcomes were number of exacerbations and hospitalisations. The telemedicine group had a lower rate of exacerbations and hospitalisations. A similar study used a smartphone for daily symptom recording and detection of exacerbations [32]. The patients were COPD GOLD level I-IV 
(IV, $\mathrm{n}=11)$ patients and participants transmitted daily symptom diaries. Symptom changes, missed diary transmissions or medical care for a respiratory problem triggered alerts and participant encounters were initiated at the time of suspected COPD exacerbations. Returns to normal breathing were also reported. Compliance was excellent, with $99.9 \%$ of 28514 possible daily diaries transmitted successfully. All 191 (2.5 per participant-year) COPD exacerbations were detected. During 148 (78\%; 1.97 per participant-year) of the 191 exacerbations, patients were hospitalised and/or administered prednisone, an antibiotic or both. This smartphone-based collection of COPD symptom diaries allowed for near-complete exacerbation identification at inception. Another study using the smartphone collected patient-reported outcomes [33]. This 4-month randomised clinical trial used clinical criteria and the EXACT PRO (Exacerbations of Chronic Pulmonary Disease Tool, Patient-Reported Outcomes) questionnaire to identify exacerbations. All patients completed a diary questionnaire on the smartphone each day and they were contacted and assessed if they appeared to be having an exacerbation. The platform used for telemedicine allowed a wide variety of functionalities and was supported by a central clinic-based call centre. A web-based application facilitated patient accessibility and interactions among professionals working at different levels of the system.

Remote healthcare technologies have been shown to reduce the number of emergency admissions to hospitals in patients with long-term conditions, including COPD. The sample included 6000 participants who had a social care needs, COPD, heart failure or diabetes. Telecare or telemedicine that relay data to nurses or doctors were installed in the participants' homes. The programme cost $£ 31$ million ( $€ 36$ million; \$51 million) and collected data for more than 2 years; however, it was cost-effective and the study concluded by stating that "The question is whether these outcomes will remain and continue in the long term in clinical practice" [34].

Integrating a tailored telehealth self-management application for COPD patients into primary care was conducted as a pilot study in 2014. Changes in reimbursement compelled Dutch primary-care practices to apply a disease-management approach for patients with COPD. This approach includes individual patient consultations with a nurse, who coaches patients in COPD management. Adding a web-based self-management support application that assessed patients' health status, the impact on the organisation of care and the level of application use and appreciation moved the standard care into the realm of telemedicine. Results suggest that it is possible to integrate a web-based COPD self-management application into the current primary-care disease-management process. The pilot study also revealed opportunities to improve the application and reports, in order to increase technology use and appreciation [35].

Another perspective on the limitations of measuring the effectiveness of telemedicine in caring for patients with COPD was reported by PINNOCK et al. [36] in a randomised trial study of 256 patients. Patients who began the study with a history of exacerbations and readmissions did not experience a significant difference in postponed readmissions or improved quality of life when compared with the conventional self-monitoring comparison group. They suggest that "the positive effect of telemonitoring seen in previous trials could be due to enhancement of the underpinning clinical service rather than the telemonitoring communication" [36].

Several clinical trials reported on integrated care for patients with COPD and multiple comorbidities (PICCOPD+). A case manager provided weekly phone contact to the patients. Short-term telehealth follow-up after hospital discharge for COPD involved placing telemedicine equipment at the patient's home. Each morning the COPD Assessment Test questionnaire was assessed using the telemedicine equipment as well as recorded extra use of COPD relief medications. Also telespirometry and teleoximetry at 4 and 12 weeks were performed in the home. When a clinical worsening was detected, the patient was contacted and asked to perform ad hoc telespirometry and teleoximetry and to send the data to the telehealth centre. Advanced e-Health for COPD in Colorado (USA) involved telehealthcare with an APRN and usual homecare. Nurse teleconsultations with discharged COPD patients reduced the numbers of readmissions. Telemonitoring video conferences using APRNs successfully monitored COPD patient care. 
Additional studies have examined the importance of cognitive function in patients with COPD who receive telemedicine [37]. Cognitive function, especially memory and attention, is more impaired in COPD patients than in healthy controls [38]. A recent study published by SCHOU et al. [38], from Denmark, investigated whether cognitive performance in patients with severe COPD and a mildto-moderate exacerbation is better after telemedicine-based treatment ("virtual admission") compared with conventional hospital admission. The study included patients admitted to a medical emergency department with COPD exacerbations. Within 24 hours after admission, patients were randomised to continued treatment either in their own home with telemedicine or in the hospital. Patients were excluded if they had a Mini Mental State Examination (MMSE) score of less than 24 points and/or current severe psychiatric disease. The authors concluded that patients with severe COPD suffering from mild-to-moderate exacerbations were able to manage the telemedicine-based treatment despite the reduced cognitive function often seen in COPD patients and, more importantly, practitioners operating telemedicine system should be aware of COPD patients' reduced cognitive function when considering systems that need a high level of selfmanagement [39].

\section{Regions of Europe Working Together for Health}

Patients with COPD were included in Renewing Health (Regions of Europe Working Together for Health), a large scale project carried out in nine European regions targeting patients with different chronic conditions. In the regions of southern Denmark, Catalonia and central Greece, the prevention of early hospital readmissions of COPD patients were studied. The overall aim of Renewing Health was to confirm whether ICS with the support of ICT (ICS-ICT) improve quality of life and user satisfaction, enable patient empowerment and have potential for cost containment by reducing healthcare service use. The details of the telemedicine interventions and the technologies used differ between the regions and ICS assessment. Detailed information at regional levels can be found at the Renewing Health website (www. renewinghealth.eu).
The study in Catalonia, Spain, evaluated the effects of a telehealth intervention for home-based follow-up of COPD patients discharged from hospital after acute exacerbation. Eight hospitals in Barcelona participated in the study. A randomised controlled trial design was performed, which included 380 COPD patients allocated to intervention or usual care. Intervention was tailored according to the patient's clinical complexity and included: video-teleconsultations, daily remote monitoring with sensors and access to a call centre and to a web-based patient health portal. Assessment was performed at discharge and after a 3-month followup period. Economical and organisational impacts of the service were also evaluated. Assessment was based on the Model for Assessment of Telemedicine applications (MAST). The analysis of the data at cluster level is still ongoing.

A Cochrane systematic review of telehealthcare for COPD [40] was performed, including only high-quality evidence from randomised trials, and found evidence of possible quality of life benefits of telehealthcare, as well as decreased emergency department visits and hospital admissions. Most of the interventions evaluated, however, were not teleconsultation, but were a mixture of telehealthcare as part of a complex intervention that also included case management by a nurse or other interventions, which makes isolating the effect of the telemedicine component difficult.

\section{Telemedicine and cystic fibrosis}

Cystic fibrosis (CF) is a severe genetic disease which primarily affects the lungs and digestive system. In CF sufferers, a defective gene and its protein product cause the body to produce unusually thick, sticky mucus, obstructing the lungs and leading to lung infections. An estimated 70000 children and adults worldwide have CF. More than $75 \%$ of people with CF are diagnosed by the age of 2 years and, today, about half of the CF population is aged 18 years or older. In the 1950s, the life expectancy of children with CF was so low that many did not reach primary school. However, tremendous progress in the field of CF has led to dramatic improvements in the length and quality of life for patients, 
extending life expectancy into the 30s, 405 and beyond. The technology skills of these families and young adults suggest that telemedicine could be an important component of their treatment.

Children and adults with CF today have more therapy options than they have ever had before: medications to clear the mucus from airways and reduce inflammation; aerosolised antibiotics; and, recently, breakthrough genetic therapy to address the underlying cause of CF. Telemedicine is being used to track patient lung function daily in order to anticipate problems and adjust treatments, such as increasing breathing treatments or antibiotics, before they need to be hospitalised. Telemedicine has been successfully implemented in schools to continue to monitor the children.

\section{Description of telemedicine interventions for patients with CF}

A feasibility study of home telemedicine for patients with CF awaiting transplantation studied 16 terminally ill patients with CF. Videoconferencing units connected to their home televisions were used for weekly conferences that included a clinical assessment, psychological counselling and discussions with members of the multi-disciplinary team. Although there was no significant difference in psychological parameters of anxiety and depression, patients liked and valued the service and showed significant improvement in body image.

Assessing exercise capacity using telehealth for 10 adults with CF [41] demonstrated that exercise capacity assessment using the 3-min step test was feasible and accurate using remote videoconferencing. Oxyhaemoglobin saturation and heart rate were monitored accurately, although there were no significant changes over time. Participants found the system easy to use, but metronome acoustics were reported to be problematic for the clinician at the remote site. Further study of the ability to anticipate exacerbations based on exercise capacity assessment is ongoing.

When CF patients using telemedicine were compared with COPD patients also using telemedicine in the same study [42], exacerbations were detectable in both groups. However, the COPD patients were more compliant, had relatively fewer hospitalisations and reduced exacerbations. Thus, adherence appears to be an issue for CF patients [43].

A systematic review of telehealth in CF [44] found eight studies, of which seven were feasibility studies, pointing to the relative novelty of using telemedicine in CF. Significant findings in the intervention study related to increased use of antibiotics and improved spirometry stability. One challenge in assessing feasibility was the relatively high level of participant non-compliance $(43 \%$ to $63 \%$ ) with transmission of spirometry data, even though participants reported they were able to use the equipment. The use of telemedicine for patients with CF is a promising technology deserving further study.

\section{Telemedicine and pulmonary hypertension}

Pulmonary hypertension $(\mathrm{PH})$ is a rare (estimated prevalence of $15-50$ cases per million [45]) haemodynamic condition defined as an increase in mean pulmonary arterial pressure (PAP) of $25 \mathrm{mmHg}$ at rest determined by right heart catheterisation. The prevalence of $\mathrm{PH}$ is most common in patients with sickle cell disease, systemic sclerosis and HIV [46-48] and symptoms are shortness of breath, dizziness and fatigue [49]. Due to the non-specific nature of symptoms, $\mathrm{PH}$ is most frequently diagnosed when patients are in advanced stages of the disease [50].

Currently, there is no cure for $\mathrm{PH}$ and treatment is solely aimed at improving symptoms and exercise tolerance, long-term outcomes and quality of life, as well as slowing the rate of deterioration [51]. Disease management in $\mathrm{PH}$ is complex involving a range of treatment options including prevention and prompt treatment of chest infections, as well as supportive oxygen therapy and medication. As such, there is no specific telemonitoring for at home care of $\mathrm{PH}$ patients; however, support for clinical decision making has been investigated. Telemedicine assessment supporting lifestyle changes, medicines and posthospital care may alter the progression of the disease [52]. 


\section{Description of telemedicine interventions in $\mathbf{P H}$}

Diagnostic studies to obtain the final diagnosis may be remotely interpreted by the limited number of $\mathrm{PH}$ disease specialists. Complex treatment (pharmacological and non-pharmacological) regimens may be monitored by telemedicine. Therapeutic education has a major role to play in management of complex diseases. The importance of therapeutic adherence in $\mathrm{PH}$ management suggests that selfmonitoring systems could be as useful as they have been in other chronic diseases. Videoconferencing and monitoring of symptoms and signs could improve outcomes. However, more research is needed in this area, using integrated care with the support of ICT.

\section{What are the main reasons to use telemonitors for patients with CRD (and comorbidities)?}

\section{Patient}

Because of the concomitant chronic conditions, single disease-oriented clinical guidelines are not adequate for management of comorbid conditions. There is a need for patient-centred care to achieve an optimal management in terms of biomedical requirements, especially candidates for homecare and rehabilitation. Caregivers appreciated telemedicine use and contact with nurses in monitoring patients at home and patients were satisfied that telemedicine helps them feel supported after hospital discharge giving them confidence, so care is ongoing and self-management can be improved.

\section{Clinician}

Telemonitoring supported pulmonary care at home and was a prevention strategy for acute problems through low-intensity home-based services coordinated by the clinical team with the support of a specialised nurse case manager. Home-based services could be coordinated with the potential support of pulmonary nurses and oxygen services, with nurse coaching based on telemonitoring data following guidelines prepared by physicians.

\section{System}

Integrated care services with the support of telemonitoring integrates key components in improving care for patients with chronic respiratory diseases. Information sharing across the healthcare system is a crucial element to avoid duplication and extensive use of acute care resources (such as emergency rooms) in these patients.

\section{What are the limitations of telemonitors in}

\section{management of patients with chronic respiratory disease (and comorbidities)?}

\section{Patient}

It can be more difficult to develop trust of care providers who are not actually present in the home, and technology (especially for older adults) may be intimidating, so telemonitoring systems must be easy to use and not be strenuous to use. Also, using telemonitors may raise concerns for "medical consultation" privacy.

\section{Clinician}

Risk management from a distance has been a concern, often dependent on the level of training for the individual reviewing the data. Clinician-patient relationships potentially can be further complicated by communication technology, especially when the delivery system does not include visual contact with the clinician. Clinicians at a distance may have difficulty communicating if the patient develops dementia and impaired senses.

\section{System}

Telemedicine needs specific policies and procedures to address risk of liability. Technological support may be limited in rural areas so systems must be tested prior to fullscale implementation. Costs may increase as equipment changes and is updated. A question that continually impacts the system level is how calculation of cost-benefit can best be approached. Telemedicine can alter the doctor-patient relationship. Careful assessment of effectiveness, cost effectiveness and safety considerations is needed before introduction. Potential pitfalls include user interface problems, technical problems and safety concerns, such as data loss and confidentiality. 


\section{What are the challenges of telemedicine?}

A major telemedicine cost-effectiveness study was funded as the European Project, NEXES [16]. It was designed to explore the determinants of capacity building of innovative ICSICT systems across European Regions. The main objective of NEXES was to validate four ICT-enabled ICS in large-scale trials, in the setting of the chronic conditions: COPD, congestive heart failure and type II diabetes mellitus. The four integrated care systems assessed in the NEXES project were: 1) Wellness and Rehabilitation $(n=337)$; 2) Enhanced Care $(n=1340)$; 3) Home Hospitalization $(n=2404)$; and 4) Support program to remote diagnosis $(n=8139)$. These studies were conducted in three different sites, Barcelona (Spain), Athens (Greece) and Trondheim (Norway), using both randomised controlled trials and pragmatic study designs. Two ICT platforms were used to support the ICS: 1) a health information exchange platform (i.e. the "Elin" platform) in Trondheim; and 2) a health information sharing platform (i.e. Linkcare) in Barcelona, and in Athens using a service-oriented approach. The model for assessment of telemedicine applications was adopted for evaluation purposes and the NEXES project was an example of good practice. The success lay in the translation of hospital complexity to the community using ICT as a supporting tool. NEXES formulated specific strategies for implementation of these ICS at European level.

\section{Best practices to develop telemedicine as a component of an ICS}

\section{Assessment}

Screening via questionnaire can be built into the system with patients prompted to complete the data. The nurse clinician can have

\section{Challenges of telemedicine}

- Legal issues surrounding physician licensing and patient confidentiality

- Liability

- Cost

- Reimbursement access to the clinical view using an office platform screen with easily accesible past reports.

\section{Intervention}

Self-management supporting presence can be a virtual presence to respond to nonverbal cues, as well as when a patient feels the need for contact. It is critical to have a plan and to respond with evidence-based interventions when risks are highlighted. Self-monitoring can act as relapse prevention and result in patients becoming more aware of their risks and responses.

Telemonitoring and telenursing (using data on patient knowledge, symptoms and behaviour) can prevent derailment, support daily functioning, answer and monitor questions, including anticipatory guidance and the recognition of "missed" symptoms.

Care provided includes specialised information on demand via telephone or website, advice for social needs, triage for appropriate care, and self-management advice or second opinions can be obtained using data records and visuals.

Summary of the European Economic and Social Committee's opinion on telemedicine

- Telemedicine activity must be of at least equivalent quality to a traditional activity

- The patient must be able to give their free consent

- User-friendly software is essential

- Medical confidentiality must be ensured

- Resulting documents must be secure and recorded in the medical file

- Continuity of care must be ensured

\section{Lessons learned}

Systems that can operate interchangeably (e.g. technical, languages) are key factors when moving from testing a telemedicine system to practical applications on a larger scale. Although geographic aspects must be considered in the ability of systems to transmit data, the ability to communicate with other data sources must be considered. 
Prior to implementation, there should be a review of needs for clinical care, experimental data collection, different informational needs from providers and academia and, perhaps most importantly, interoperability at organisational levels.

Another challenge to consider is reimbursement in order to have financial sustainability. Consider the alignment between what reimbursement is possible and how the processes included in the telemedicine care programmes (services) can be reported congruent with reimbursement requirements. For actualisation of telemedicine by clinicians/practices, financial factors that can be used as incentives will be needed to support utilisation and sustainable adoption.

Evidence of benefits is needed to develop systems and policy changes. Strong evidence has been lacking due to heterogeneity of telemedicine studies; societal perspectives on preferring "high touch rather than high

\section{Educational questions}

1. All of the following are components of the definition of telemedicine except:

a. Advanced information and communication technologies used to transmit information electronically.

b. Patient keeps a record of self-monitored information in a daily log and brings it to the clinician at the next visit for review.

c. Electronic medical devices to support the delivery of clinical care

d. Electronic medical devices to support the delivery of professional education

e. Electronic medical devices to support the delivery of health related administrative services

2. Many patients living at home could benefit from telemedicine. Organised screening is important to identify those patients who would benefit from telemonitoring. Screening would include at a minimum the patient/family's ability to use the equipment, safety of using telemedicine in the home, and the availability of local service for sending information.
a. True
b. False

3. The European Economic and Social Committee's opinion on telemedicine includes all except:

a. The medical act must be of at least equivalent quality to a traditional act.

b. The patient must be able to read the forms

c. Medical confidentiality must be ensured

d. Resulting documents must be secure and recorded in the medical file

e. Continuity of care must be ensured

f. User-friendly software tech"; limited use of appropriate methodology to show efficacy in terms of clinical outcomes, cost containment, satisfaction of patients and potential for generalisation. Assessment models such as MAST, although not without limitations, are essential to document value.

Regulatory issues must be considered as new roles for professionals are developed. Licensing to provide care in areas with different laws can limit the ability to provide telemedicine care across borders. Structural changes in organisations who are the key stakeholders may be needed to support data sharing. Information sharing and transfer of responsibilities among levels of care and among providers must be designed into telemedicine care protocols. Novel uses of equipment and software applications have different implications at international levels, and national/regional levels. As telemedicine systems are planned and implemented, there will be a constant need to re-evaluate and investigate new options. Finally, implementing a telemedicine service requires consideration of human factors and related organisational characteristics that involve cultural, educational and motivational aspects of the patient and clinician who are at different levels of the healthcare systems.

\section{Conclusions}

The development of telemedicine has been driven partly by technological advances. Its potential role has been highlighted by the European Commission for more than a decade in order to address the issues of an ageing population, chronic conditions and rising healthcare costs. The European Commission's action plan for a European "eHealth area" states several goals to its member states and has set a deadline for the widespread deployment of telemedicine service of 2020. A recent consultation on an e-Health action plan for 2012-2020 reported that there was a need to support deployment of research results and that more flexible financing mechanisms for research and innovation needed to be provided. The research should outline the benefits and costs, and the effectiveness and usefulness of telemedicine solutions. Studies of factors that could limit adoption of systems supported by ICT are also needed [53]. A recent paper provided a literature review of the value of telemedicine in the management of five 
common chronic diseases (asthma, COPD, diabetes, heart failure and hypertension) over the past 20 years [4]. The review concluded that the evidence base for the value of telemedicine in managing chronic diseases is, on the whole, weak and contradictory, and may be related to the fact that most studies were conducted over a period of 6 months or less; it is challenging to show a change in chronic disease management over such a short time period. Nevertheless, studying telemedicine solutions among patients with chronic respiratory diseases demonstrates high levels of acceptance of these applications, such as appointment booking, prescription renewal and access to information (laboratory test results, educational resources, etc.), and a challenge in supporting the comparative effectiveness of solutions directly related to medical care (communication with healthcare providers, disease monitoring, patient outcomes, etc.) [54].
Using telemedicine, patient satisfaction is high, while provider satisfaction is mixed. Research on clinical efficacy has mixed results. Unequivocal evidence of the relative efficacy or cost-effectiveness of telemedicine has not yet materialised despite many years of effort across perhaps 1500 individual studies. Nevertheless, the application of telemedicine in practice has been useful when best practices are followed in design and implementation with a system change approach. Considering the value of improved quality of life and productivity, there have been great returns on investments in applying health technology to respiratory care.

\section{Acknowledgements}

We are grateful to Dr. Josep Roca of the Hospital Clinic, Barcelona Spain for his assistance in preparation of this article.

\section{References}

1. World Health Organization. Preparing a health care workforce for the 21st century: the challenge of chronic conditions. www.who.int/chp/knowledge/ publications/workforce_report.pd? $\mathrm{Pua}=1$

2. Information and Communication Technology. http:// dictionary.reference.com/browse/Information

3. Center for Medicare \& Medicaid Services. www. medicaid.gov/Medicaid-CHIP-Program-Information/ By-Topics/Delivery-Systems/Telemedicine.html

4. Wootton R. Twenty years of telemedicine in chronic disease management-an evidence synthesis. J Telemed Telecare 2012; 18: 211-220.

5. Barr VJ1, Robinson S, Marin-Link B, et al. The Expanded Chronic Care Model: An Integration of Concepts and Strategies from Population Health Promotion and the Chronic Care Model. Hosp Q 2003; 7: 73-82.

6. Wagner EH, Austin BT, Davis C, et al. Improving chronic illness care: translating evidence into action. Health Aff 2001; 20: 64-78.

7. Hernandez C, Jansa M, Vidal M, et al. The burden of chronic disorders on hospital admissions prompts the need for new modalities of care: A cross-sectional analysis in a tertiary hospital. QJM 2009; 102: 193-202.

8. Petitte TM, Narsavage GL, Chen Y-J, et al. Feasibility study: home telemonitoring for patients with lung cancer in a mountainous rural area. Oncol Nurs Forum 2014; 41: 153-161.

9. Casas A, Troosters T, Garcia-Aymerich J, et al. Integrated care prevents hospitalisations for exacerbations in COPD patients. Eur Respir J 2006; 28: 123-130.

10. Hernandez C, Casas A, Escarrabill J, et al. Home hospitalisation of exacerbated chronic obstructive pulmonary disease patients. Eur Respir J 2003; 21: 58-67.

11. Stead LF, Hartmann-Boyce J, Perera R, et al. Telephone counselling for smoking cessation. Cochrane Database Syst Rev 2013; 8: CDoo2850.
12. Burgos F, Disdier C, de Santamaria EL, et al. Telemedicine enhances quality of forced spirometry in primary care. Eur Respir J 2012; 39: 1313-1318.

13. National Institutes of Health. Health Topics: Chest CT Scan www.nhlbi.nih.gov/health/health-topics/ topics/cct/\#

14. Rangasamy $M$, Balasubramaniam A, Krishnarajan D, et al. Role of telemedicine in health care system: a review. Int J Recent Adv Pharma Res 2011; 2: 1-10.

15. Rutledge CM, Haney $\mathrm{T}$, Bordelon $\mathrm{M}$, et al. Telehealth: preparing advanced practice nurses to address healthcare needs in rural and underserved popula tions. Int J Nurs Educ Scholarsh 2014; 11.

16. Schlachta-Fairchild L, Varghese SB, et al. Telehealth and telenursing are live: APN policy and practice implications. J Nurse Practitioner 2010; 6: 98-106.

17. Roca JGH, Grimsmo A, Meya M, et al. NEXES: supporting healthier and independent living for chronic patients and elderly: final report. www. nexeshealth.eu/media/pdf/nexes_final_report.pdf

18. Mallow JA, Theeke LA, Barnes ER, et al. Using $\mathrm{mHealth}$ tools to improve rural diabetes care guided by the chronic care model. Online J Rural Nurs Health Care 2014; 14: 43-65.

19. May C, Harrison R, MacFarlane $A$, et al. Understanding the normalization of telemedicine services through qualitative evaluation. J Am Med Inform Assoc 2003; 10: 596-604.

20. Zanaboni P, Lettieri E. Institutionalizing telemedicine applications: the challenge of legitimizing decisionmaking. J Med Internet Res 2011; 13: e72.

21. Mallol J, Crane J, von Mutius E, et al. The International study of asthma and allergies in childhood (ISAAC) phase three: a global synthesis. Allergologia et immunopathologia 2013; 41: 73-85.

22. Sverrild A, Ulrik Boedtger $U$, et al. Diagnosing asthma in a real life setting-which test to use? Am J Respir Crit Care Med; 2014: A1368.

23. Nadeau GA, Samji I, Walters RD, et al. Asthma control in cities of developing countries: results of a five-city survey. Afr J Respir Med 2014; 9: 18-23.

\section{Suggested} answers

1. $b$

2. $a$

3. b 
24. Centers for Disease Control and Prevention. Asthma Facts: CDC's National Asthma Control Program Grantees. Centers for Disease Control and Prevention, Atlanta 2013.

25. National Heart, Lung and Blood Institute Expert Panel Report 3: Guidelines for the Diagnosis and Management of Asthma. National Institutes of Health, Bethesda 2007.

26. Eberhart NK, Sherbourne CD, Edelen MO, et al. Development of a measure of asthma-specific quality of life among adults. Qual Life Res 2014; 23: 837-848.

27. McLean S, Chandler D, Nurmatov $U$, et al. Telehealthcare for asthma. CMAJ 2011; 183: E733-E742.

28. Ryan D, Price D, Musgrave SD, et al. Clinical and cost effectiveness of mobile phone supported self monitoring of asthma: multicentre randomised controlled trial. BMJ 2012; 344: e1756.

29. McKinstry B, Pinnock, H, Sheikh, A. Telemedicine for management of patients with COPD. Lancet 2009; 374: 672-673.

30. Vitacca M, Bianchi L, Guerra A, et al. Tele-assistance in chronic respiratory failure patients: a randomised clinical trial. Eur Respir J 2009; 33: 411-418.

31. Pedone C, Chiurco D, Scarlata S, et al. Efficacy of multiparametric telemonitoring on respiratory outcomes in elderly people with COPD: a randomized controlled trial. BMC Health Serv Res 2013; 13: 82.

32. Johnston NW, Lambert K, Hussack P, et al. Detection of COPD exacerbations and compliance with patientreported daily symptom diaries using a smartphonebased information system BlackBerry-based symptom diaries for COPD patients. Chest 2013; 144: 507-514.

33. Halpin D, Laing-Morton T, Spedding S, et al. A randomised controlled trial of the effect of automated interactive calling combined with a health risk forecast on frequency and severity of exacerbations of COPD assessed clinically and using EXACT PRO Prim Care Respir J 2011; 20: 324-331.

34. Vickers H: Telemedicine may cut emergency admissions for COPD, vol. 342; 2011.

35. Purcell R, Mclnnes S, Halcomb EJ. Telemonitoring can assist in managing cardiovascular disease in primary care: a systematic review of systematic reviews. BMC family practice 2014; 15: 43.

36. Pinnock $\mathrm{H}$, Hanley J, McCloughan L, et al. Effectiveness of telemonitoring integrated into existing clinical services on hospital admission for exacerbation of chronic obstructive pulmonary disease: researcher blind, multicentre, randomised controlled trial. BMJ 2013; 347: 66070 .

37. McLean S, Nurmatov U Liu JL, et al. Telehealthcare for chronic obstructive pulmonary disease. Cochrane Database Syst Rev 2011; 7: CDoo7718.

38. Schou L, Østergaard B, et al. Cognitive dysfunction in patients with chronic obstructive pulmonary diseaseA systematic review. Respir Med 2012; 106: 1071-1081.
39. Schou L, Østergaard B, Rasmussen LS, et al. Telemedicine-based treatment versus hospitalization in patients with severe chronic obstructive pulmonary disease and exacerbation: effect on cognitive function. A randomized clinical trial. Telemed J $E$ Health 2014; 20: 640-646.

40. McLean S, Protti D, Sheikh A. Telehealthcare for long term conditions. BMJ 2011; 342: d120.

41. Cox NS, Alison JA, Button BM, et al. Assessing exercise capacity using telehealth: a feasibility study in adults with cystic fibrosis. Respir Care 2013; 58: 286-290.

42. Jarad NA, Sund ZM. Telemonitoring in chronic obstructive airway disease and adult patients with cystic fibrosis. J Telemed Telecare 2011; 17: 127-132.

43. Grzincich G, Gagliardini R, Bossi A, et al. Evaluation of a home telemonitoring service for adult patients with cystic fibrosis: a pilot study. J Telemed Telecare 2010; 16: 359-362.

44. Cox NS, Alison JA, Rasekaba T, et al. Telehealth in cystic fibrosis: a systematic review. Telemed Telecare 2012; 18: 72-78.

45. Peacock AJ, Murphy NF, McMurray JJV, et al. An epidemiological study of pulmonary arterial hypertension. Eur Respir J 2007; 30: 104-109.

46. Gladwin MT, Sachdev V, Jison ML, et al. Pulmonary hypertension as a risk factor for death in patients with sickle cell disease. N EnglJ Med 2004; 350: 886-895.

47. Proudman S, Stevens W, Sahhar I, et al. Pulmonary arterial hypertension in systemic sclerosis: the need for early detection and treatment. Intern Med J 2007; 37: $485-494$

48. Sitbon O, Lascoux-Combe C, Delfraissy J-F, et al. Prevalence of HIV-related pulmonary arterial hypertension in the current antiretroviral therapy era. Am J Respir Crit Care Med 2008; 177: 108-113.

49. Badesch DB, Raskob GE, Elliott CG, et al. Pulmonary arterial hypertension baseline characteristics from the REVEAL Registry. Chest 2010; 137: 376-387.

50. Humbert M, Sitbon O, Chaouat A, et al. Pulmonary arterial hypertension in France: results from a national registry. Am J Respir Crit Care Med 2006; 173: 1023-1030.

51. Galie N, Hoeper M, Humbert M, et al. Guidelines for the diagnosis and treatment of pulmonary hypertension. Eur Respir J 2009; 34: 1219-1263.

52. Sanchez O, Humbert M, Sitbon O, et al. [Guidelines for the diagnosis and treatment of pulmonary hypertension.] Rev Mal Respir 2010; 27: 141-150.

53. Gagnon M-P, Desmartis $M$, Labrecque $M$, et al. Systematic review of factors influencing the adoption of information and communication technologies by healthcare professionals. J Med Syst 2012; 36:1, 241-277.

54. Duplaga M. The acceptance of e-health solutions among patients with chronic respiratory conditions. Telemed e-Health 2013; 19: 683-691. 